\title{
Strong coupling in a two-dimensional semiconductor/noble metal multilayer platform
}

\author{
Vasilios Karanikolas $\odot,{ }^{1, *}$ Ioannis Thanopulos $\odot,{ }^{2}$ and Emmanuel Paspalakis $\oplus^{2}$ \\ ${ }^{1}$ International Center for Young Scientists (ICYS), National Institute for Materials Science (NIMS) 1-1 Namiki, \\ Tsukuba, Ibaraki 305-0044, Japan \\ ${ }^{2}$ Materials Science Department, School of Natural Sciences, University of Patras, Patras 265 04, Greece
}

(Received 14 May 2020; accepted 1 July 2020; published 24 July 2020)

\begin{abstract}
We investigate the Purcell factor and emission spectrum of a two-level quantum emitter placed near a planar multilayer nanostructure composed of an ultrathin dielectric or noble metal film between a pair of two-dimensional (2D) layers of transition-metal dichalcogenides. We focus on the 2D semiconductor layer of $\mathrm{WS}_{2}$. Experimental values for the dielectric permittivity of the $\mathrm{WS}_{2}$ and the $\mathrm{Au}, \mathrm{Ag}, \mathrm{Cu}$, and $\mathrm{Al}$ noble metals are used for computing their optical response. The Purcell factor of the quantum emitter near such a nanostructure takes values above $10^{4}$. The emission spectrum of the quantum emitter features profound Rabi splittings at the visible part of the spectrum at room temperature indicating the emergence of the strong light-matter coupling regime. The emission spectrum of the quantum emitter is strongly influenced by the dielectric permittivity of the host medium and the material quality of the noble metal. Our research paves the way for applications in quantum technologies and biosensors.
\end{abstract}

DOI: 10.1103/PhysRevResearch.2.033141

\section{INTRODUCTION}

The interaction between quantum emitters (QEs) and nanostructures is of fundamental interest to a vast amount of photonic applications. The interaction between a single QE and light is, in general, weak. One path to overcome this limitation is through the excitation of the hybrid modes comprised of the free electrons of a metal and the electromagnetic field, the surface plasmon modes. These modes are confined in the interface between a metal and a dielectric while they propagate along that interface [1]. Applications using coupled QEs with plasmonic nanostructures, such as biosensors, Raman-scattering, and light emitting and harvesting devices, are in the spotlight due to their importance for the economy and the environment. These types of devices operate in the weak coupling regime, where the light emission takes place incoherently. On the opposite side of the interaction regime we have the strong coupling, where coherent exchange of energy between the QE and its environment takes place [2-4]; this effect is of fundamental importance for the quantum technologies, quantum computing, switching, and single photon emitter applications [5-7].

Towards the realization of quantum technologies, one of the main limiting factors is how to preserve quantum coherence over repeated cycles of information exchange between the qubits [7]. Operating in the strong coupling regime can improve this limitation. Recently, complex nanostructures

\footnotetext{
*KARANIKOLAS.Vasileios@nims.go.jp

Published by the American Physical Society under the terms of the Creative Commons Attribution 4.0 International license. Further distribution of this work must maintain attribution to the author(s) and the published article's title, journal citation, and DOI.
}

have been fabricated in order to achieve strong coupling interaction regime between QEs and a nanostructured photonic environment, such as photonic crystals [8], or the gaps created randomly between nanocubes and back reflectors $[9,10]$.

Usually strong coupling regime is achieved when a $\mathrm{QE}$ is placed within a cavity, dielectric or metallic. The dielectric cavities provide huge mode volumes, but they are limited due to the diffraction effects. In addition, when multiple QEs are placed in such cavities the interaction strength drops very fast. In comparison, a metallic cavity can confine the light in subdiffraction dimensions, down to nanometer dimension, by using the surface plasmon modes [1]. Noble metals are routinely used for photonics applications in the visible part of the spectrum, although they have a major drawback when bulk nanostructures are considered, since in such case the material losses seriously deteriorate the efficiency of the applications [11]. However, new fabrication methods are currently applied in order to produce pristine ultrathin noble metal films $[12,13]$ with little material losses.

Recently, a new family of materials, the transitionmetal dichalcogenides (TMDs), has attracted a significant amount of research, both experimentally and theoretically [14-17]. These two-dimensional semiconducting materials show strong absorption and emission features in the visible part of the spectrum. Furthermore, when multiple layers of TMD materials are considered, they can support waveguide exciton polariton modes, which are hybrid modes of the electromagnetic field and the excitons of the semiconducting material [18]. It has been predicted that the exciton polariton modes will be supported even by a single TMD layer [19] provided it is a pristine monolayer.

The investigation of the light-matter interaction between localized sources and a nanostructured environment is under intensive experimental and theoretical investigation both in the weak and in the strong coupling regimes. 
The relaxation rate of a $\mathrm{QE}$ has been investigated close to various nanostructures, such as metallic [20-29] and graphene [30-36] nanostructures of varying dimensionality, carbon nanotubes [35,37,38] and TMD materials [19,39-41].

Recently, we showed that when a QE is placed near a $\mathrm{WS}_{2}$ layer with an Au substrate, its emission properties are modified and the light-matter interaction strength lies in the strong coupling regime, as indicated by the Rabi splitting in the emission spectrum of about $100 \mathrm{meV}$ [40]. In this work we extend our previous study by considering a QE near a nanostructure composed of a thin film made by one of the noble metals $\mathrm{Au}, \mathrm{Ag}, \mathrm{Cu}$, and $\mathrm{Al}$ or by a homogeneous dielectric, sandwiched between a pair of $\mathrm{WS}_{2}$ layers. In the $\mathrm{WS}_{2} /$ metal film/ $\mathrm{WS}_{2}$ nanostructures, the hybridization of the excitons of the $\mathrm{WS}_{2}$ layers with the plasmon modes of the thin film strongly modifies the emission properties of the nearby QE. As a result, a distinct emission pattern is observed, where a pair of splittings may lead to the emergence of a triple-peaked emission spectrum. Furthermore, the 2D semiconductor/noble metal multilayer nanostructure is tunable with respect to the dielectric permittivity of the host medium and the exact positioning of the QE.

In this paper we investigate the interaction between a $\mathrm{QE}$ and a multilayer planar nanostructure composed from a pair of $\mathrm{WS}_{2}$ layers with a thin metallic or dielectric film in between. We start in Sec. II by giving the theoretical description of the spontaneous emission (SE) of a QE in the weak and strong coupling regimes. Specifically, we present the calculation of the electromagnetic Green's tensor used in determining the Purcell factor and the SE spectrum of the QE. In Sec. III we discuss the exciton-plasmon dispersion relation and its influence on the SE of a QE near the nanostructures under study. The most commonly used noble metals, $\mathrm{Au}, \mathrm{Ag}, \mathrm{Cu}$, and $\mathrm{Al}$, are considered. We find that close to the emission energy of the exciton $A$ of the $\mathrm{WS}_{2}$, the $\mathrm{Au}$ and $\mathrm{Cu}$ have the best ratio of the Rabi splitting to the emission energy considered, indicating that the interaction strength could be even in the ultrastrong coupling regime, while the $\mathrm{Al}$ has the worst such ratio. In the last section we summarize our findings.

\section{MATERIAL PARAMETERS AND THEORETICAL PART}

The nanostructure under consideration is shown in Fig. 1, where a thin film is sandwiched between a pair of $\mathrm{WS}_{2}$ layers. Dielectric and noble metal materials are considered for composing the thin film. We now present the experimentally measured dielectric permittivities of the $\mathrm{WS}_{2}$ and the noble metals ( $\mathrm{Au}, \mathrm{Ag}, \mathrm{Cu}, \mathrm{Al}$ ), and the main expressions for calculating the Purcell factor and SE spectrum of a QE in the presence of the multilayer geometry.

In Fig. 2(a) we present the dielectric permittivity of a $\mathrm{WS}_{2}$ layer extracted from experimental data by using the expression

$$
\varepsilon_{\mathrm{WS}_{2}}(\omega)=\varepsilon_{\infty}+\sum_{i=1}^{9} \frac{f_{i}}{\omega_{i}^{2}-\omega^{2}-i \omega \gamma_{i}},
$$

where the parameters in the above equation are given in Refs. [42,43]. In Fig. 2(a) we observe that the real part of

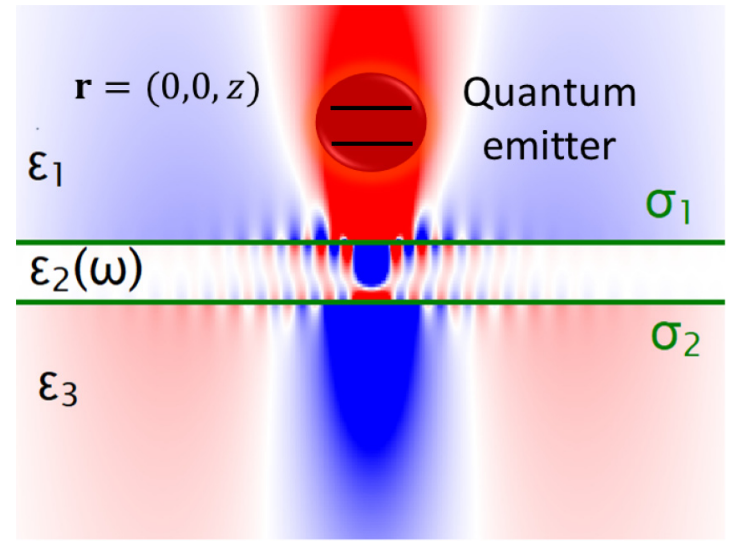

FIG. 1. A schematic representation of the system under investigation: A QE near two $\mathrm{WS}_{2}$ layers with a thin film of either a constant dielectric or a noble metal in between. The $x y$ plane is parallel to the planar nanostructure and the $z$ axis is perpendicular to it.

the $\varepsilon_{\mathrm{WS}_{2}}$ is close to zero and its imaginary part is sharply peaked at the energy of the exciton $A, \hbar \omega=2.02 \mathrm{eV}$. Here, we describe the optical response of the $\mathrm{WS}_{2}$ material through its surface conductivity, which is given by the expression $\sigma_{2 D}=$ $i \varepsilon_{0} \omega d\left(\varepsilon_{\mathrm{WS}_{2}}(\omega)-1\right)$, where $d=0.618 \mathrm{~nm}$ is the thickness of the $\mathrm{WS}_{2}$ layer. We note that the $\mathrm{WS}_{2}$ layer does not support any confined modes, connected to exciton-polariton surface modes, since at all energies around the energy of exciton $A$, $\operatorname{Re}\left[\varepsilon_{\mathrm{WS}_{2}}\right] \geqslant 0$ is valid. Thus, in case of a single $\mathrm{WS}_{2}$ monolayer, there is no transverse magnetic (TM) exciton-polariton mode. In these energies only transverse electric (TE) modes exist, which are rather loosely confined to the $\mathrm{WS}_{2}$ layer and essentially operate as radiative modes [19]. The TE waveguide modes emerge when thick TMD layers are considered [44].

In Fig. 2(b) we present the dielectric permittivities of the $\mathrm{Au}, \mathrm{Ag}, \mathrm{Cu}$, and $\mathrm{Al}$ noble metals, which determine their optical response in the energy range around the exciton $A$ of the $\mathrm{WS}_{2}$ layer $[45,46]$. The imaginary part of the dielectric permittivity is related to the material losses; the higher its value the lossier the material. Aluminum has the worst optical response among these materials in the range of energies around the exciton $A$ of the $\mathrm{WS}_{2}$. We note that in Ref. [46], novel fabrication methods for developing pristine thin noble metal films with improved optical response are presented.

A QE, which is described as a two-level system, interacts with its environment through the electromagnetic field. Thus, the excited state relaxes to the ground state by emitting a photon or exciting any of the dressed states supported by its environment [47], which in our case are the hybridized modes of the surface plasmons supported by the noble metals and the excitons of the $\mathrm{WS}_{2}$ layer, called plexcitons $[43,48]$. This relaxation process is characteristic when the $\mathrm{QE} /$ nanostructure interaction is in the weak coupling regime. In such a case, by applying Fermi's golden rule, the expression for the relaxation rate $\Gamma(\mathbf{r}, \omega)=\frac{2 \omega^{2} \mu^{2}}{\hbar \varepsilon_{0} c^{2}} \hat{\mathbf{n}} \cdot \operatorname{Im} \mathfrak{G}(\mathbf{r}, \mathbf{r}, \omega) \cdot \hat{\mathbf{n}}$ is obtained, where $\hat{\mathbf{n}}$ is a unit vector along the direction of the transition dipole moment $\mu$, and $\mathfrak{G}(\mathbf{r}, \mathbf{s}, \omega)$ is the electromagnetic Green's tensor, representing the response of the geometry under consideration to a pointlike dipole excitation. 


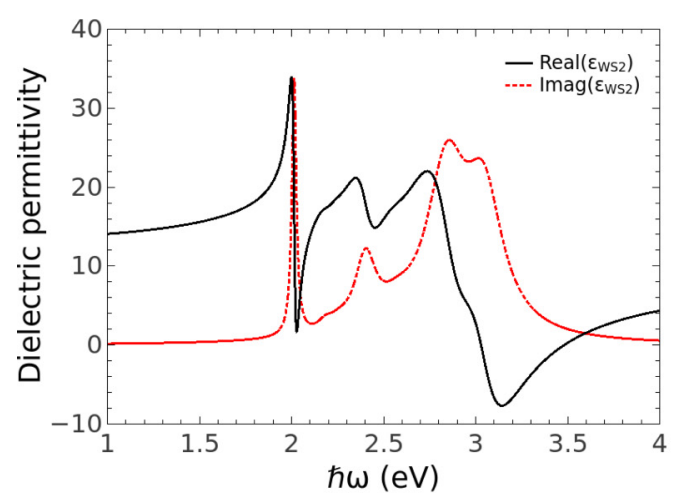

(a)

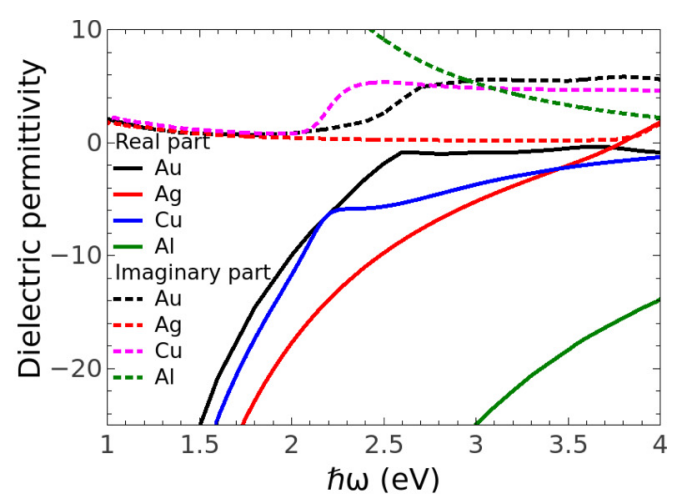

(b)

FIG. 2. (a) Real and imaginary parts of the experimental value of the dielectric permittivity $\varepsilon_{\mathrm{WS}_{2}}(\omega)$ from Ref. [42]. (b) Real and imaginary parts of the experimental value of the dielectric permittivity of the noble metals $\varepsilon_{i}(\omega), i=\mathrm{Au}, \mathrm{Ag}, \mathrm{Cu}$, and $\mathrm{Al}$.

The multilayer planar geometry is embedded in a constant dielectric permittivity medium $\varepsilon_{1}=\varepsilon_{3}$, as shown in Fig. 1. It consists of two $\mathrm{WS}_{2}$ layers with a thin film between them. The thin film has thickness $D$ and dielectric permittivity $\varepsilon_{2}$ and the two $\mathrm{WS}_{2}$ layers are considered as infinitely thin with dielectric permittivity $\varepsilon_{\mathrm{WS}_{2}}(\omega)$ and surface conductivity $\sigma_{1}=\sigma_{3}=\sigma_{2 D}$. All layers are of infinite extent in the $x y$ plane and the $z$ axis is perpendicular to the surface of each layer.

The method of scattering superposition is used [49-51] for the calculation of the electromagnetic Green's tensor. In this approach the Green's tensor splits into two parts:

$$
\mathfrak{G}(\mathbf{r}, \mathbf{s}, \omega)=\mathfrak{G}_{h}(\mathbf{r}, \mathbf{s}, \omega)+\mathfrak{G}_{s}(\mathbf{r}, \mathbf{s}, \omega) .
$$

In the above equation, $\mathfrak{G}_{h}(\mathbf{r}, \mathbf{s}, \omega)$ is the homogeneous part that accounts for direct interaction between the source and target points at $\mathbf{s}$ and $\mathbf{r}$, respectively, and is nonzero when both points are in the same media, and $\mathfrak{G}_{s}(\mathbf{r}, \mathbf{s}, \omega)$ is the scattering part, which is always present and accounts for the multiple reflections and transmissions taking place at the interfaces.

The general form of the scattering part of the Green's tensor has the form:

$$
\begin{aligned}
\mathfrak{G}_{s}(\mathbf{r}, \mathbf{s}, \omega)= & \frac{i}{8 \pi^{2}} \int d^{2} k_{s} \frac{1}{k_{z i} k_{s}^{2}} \sum_{T} R_{T}^{ \pm(i j) \pm} \mathbf{T}\left(\mathbf{k}_{s}, \pm k_{z i}, \mathbf{r}\right) \\
& \otimes \mathbf{T}^{*}\left(\mathbf{k}_{s}, \pm k_{z j}, \mathbf{s}\right)
\end{aligned}
$$

for $i, j=1,2,3$ denoting the homogeneous material above the upper $\mathrm{WS}_{2}$ layer, the thin in-between slab, and the homogeneous material below the lower $\mathrm{WS}_{2}$ layer, respectively, with $k_{z i}=\sqrt{k_{i}^{2}-k_{s}^{2}}$, while $k_{s}$ being the in-plane wave vector. We note that in Eq. (3) a summation is implied for each pair of \pm indices. These indices relate to the direction of propagation of the electromagnetic modes: the first index denotes the target point and the second the position of the QE. Also the summation over $\mathbf{T}$ takes place over the $\mathbf{M}$ and $\mathbf{N}$ modes, which are related to the transverse electric and transverse magnetic modes, respectively. The explicit form of $\mathbf{M}$ and $\mathbf{N}$ is given in Ref. [50]. We note that for the planar geometries there are no hybrid modes. The boundary conditions imposed on the system of multilayers are the continuity condition and the radiation condition. The first condition is given by continuity equations at each interface:

$$
\begin{aligned}
& \hat{\mathbf{z}} \times {\left.\left[\mathfrak{G}^{(i j)}(\mathbf{r}, \mathbf{s}, \omega)-\mathfrak{G}^{((i+1) j)}(\mathbf{r}, \mathbf{s}, \omega)\right]\right|_{z=z_{i}}=0, } \\
& \hat{\mathbf{z}} \times\left.\left[\nabla \times \mathfrak{G}^{(i j)}(\mathbf{r}, \mathbf{s}, \omega)-\nabla \times \mathfrak{G}^{((i+1) j)}(\mathbf{r}, \mathbf{s}, \omega)\right]\right|_{z=z_{i}} \\
&=-i \frac{4 \pi}{c} k_{0} \sigma \hat{z} \times \hat{z} \times \mathfrak{G}^{((i+1) j)}(\mathbf{r}, \mathbf{s}, \omega),
\end{aligned}
$$

where $z_{i}=0, D$ and $\sigma$ is the surface conductivity of the two-dimensional material, which in our case is the surface conductivity of the $\mathrm{WS}_{2}$ layer given by Eq. (2) [19].

By applying these boundary equations, an inhomogeneous system of four equations is defined with the four generalized $R_{M(N)}^{ \pm(i j) \pm}$ coefficients as unknown quantities. These coefficients are sufficient to uniquely determine the problem under consideration through the exact computation of the scattering part of the Green's tensor. In order to obtain these generalized coefficients, a matrix equation is solved, which has the form

$$
\Delta_{M(N)} \cdot \boldsymbol{R}_{M(N)}^{ \pm(i j) \pm}=\boldsymbol{V}_{M(N)}^{ \pm(i j) \pm}
$$

with $\Delta_{M(N)}$ being the characteristic matrix of the system of equations from the boundary conditions at the interfaces, $\boldsymbol{R}^{ \pm(i j) \pm}$ being the column of the generalized coefficients $R_{M(N)}^{ \pm(i j) \pm}$ and $\boldsymbol{V}^{ \pm(i j) \pm}$ being the free-terms vector, whose components are given by the homogeneous part of the Green's tensor.

By applying the boundary conditions at the $z=0$ and $z=D$ we obtain the characteristic matrices for the TE and TM polarizations. Assuming that the transition dipole moment of the $\mathrm{QE}$ is along the $z$ axis, the highest modification in the relaxation rate is along the $z$ direction due to the excitation of the TM modes only; thus, below, we focus on these modes

$$
\Delta_{N}=\left(\begin{array}{cccc}
\frac{k_{z 1}}{k_{1}} e^{i k_{z 1} D} & -\frac{k_{22}}{k_{2}} e^{i k_{z 2} D} & \frac{k_{z 2}}{k_{2}} e^{-i k_{z 2} D} & 0 \\
k_{1} e^{i k_{z 1} D} & -\left(k_{2}-\alpha k_{z 2} / k_{2}\right) e^{i k_{22} D} & -\left(k_{2}+\alpha k_{z 2} / k_{2}\right) e^{-i k_{z 2} D} & 0 \\
0 & \frac{k_{z 2}}{k_{2}} & -\frac{k_{z 2}}{k_{2}} & \frac{k_{z 3}}{k_{3}} \\
0 & k_{2} & k_{2} & -\left(k_{3}+\alpha k_{z 3} / k_{3}\right)
\end{array}\right) \text {, }
$$


where $\alpha=4 \pi \sigma_{\mathrm{WS}_{2}} \omega / c^{2}$, the generalized coefficients have the form $\mathbf{R}_{N}^{11}=\left(R_{N}^{+(11)-}, \quad R_{N}^{+(21)-}, R_{N}^{-(21)-}, R_{N}^{-(31)-}\right)$ and the free-terms vectors are given by $\mathbf{V}_{N}^{11}=\left(\frac{k_{z 1}}{k_{1}} e^{-i k_{z 1} d}\right.$, $\left.k_{1} e^{-i k_{z 1} d}, 0,0\right)$.

We consider a $\mathrm{QE}$ placed at $\mathbf{r}=(0,0, z)$ with a $z$-oriented transition dipole moment; we are mainly interested in the influence of the multilayer nanostructure to the relaxation of the QE. We now introduce the Purcell factor $\tilde{\Gamma}_{z}(\omega, \mathbf{r})=$ $\frac{\Gamma_{z}(\omega, \mathbf{r})}{\Gamma_{0}(\omega)}$, a quantity that shows how much the relaxation rate of the QE is enhanced or inhibited in the presence of the multilayer geometry; $\Gamma_{0}(\omega)$ is the free-space relaxation rate of the QE, given by $\Gamma_{0}(\omega)=\omega^{3} \mu^{2} / 3 \pi c^{3} \hbar \varepsilon_{0}$. The Purcell factor is given by the expression

$$
\tilde{\Gamma}_{z}(\omega, \mathbf{r})=\sqrt{\varepsilon_{1}}+\frac{3 c}{2 \omega} \operatorname{Im}\left(i \int_{0}^{\infty} d k_{s} \frac{k_{s}^{3}}{k_{z 1} k_{1}^{2}} R_{N}^{+11-} e^{2 i k_{z 1} z}\right),
$$

with $R_{N}^{+11-}$ being the generalized Fresnel coefficient for the reflection from the interface located at $D$, where in our case a $\mathrm{WS}_{2}$ layer is placed. We again note that the above expressions are used in case the $\mathrm{QE} /$ nanostructure interaction lies in the weak coupling regime.

In case of the strong coupling regime a different approach is needed, in which the description of the light-matter interaction between the $\mathrm{QE}$ and the nanostructure requires the spectral density $J\left(\omega_{1}, \omega, \mathbf{r}\right)$ of the electromagnetic continuum of modes as modified by the presence of the nanostructure. This reads

$$
J\left(\omega_{1}, \omega, \mathbf{r}\right)=\frac{\Gamma_{0}\left(\omega_{1}\right)}{2 \pi} \tilde{\Gamma}_{z}(\omega, \mathbf{r})\left(\frac{\omega}{\omega_{1}}\right)^{3},
$$

where $\omega_{1}$ is the energy difference between the ground and the excited states of the QE, $\Gamma_{0}\left(\omega_{1}\right)$ is the relaxation rate of the QE in free space and $\tilde{\Gamma}_{z}$ is the Purcell factor given by Eq. (7). We observe that the higher the Purcell factor the stronger the coupling of the QE with its photonic environment. Also, the value of the QE transition dipole moment is very important for approaching the strong coupling limit, since the higher its value, the less enhancement of the SE rate by the corresponding Purcell factor is required in order to achieve this [2]. Lastly, the relaxation spectrum of the $\mathrm{QE}$ is given by $[20,22,36,40]$

$$
S(\omega, \mathbf{r})=\frac{1}{2 \pi}\left|\frac{\frac{\mu^{2} \omega^{2}}{\varepsilon_{0} c^{2}} \hat{\mathbf{n}} \cdot \mathfrak{G}\left(\omega, \mathbf{r}, \mathbf{r}_{d}\right)}{\omega_{1}-\omega-\int_{0}^{\infty} d \omega^{\prime} J\left(\omega_{1}, \omega^{\prime}, \mathbf{r}\right) \frac{1}{\omega^{\prime}-\omega}}\right|^{2},
$$

with $\mathbf{r}_{d}$ and $\mathbf{r}$ being the positions of the signal detection and the position of the QE, respectively, and $\omega$ stands for the emission frequency.

\section{RESULTS AND DISCUSSION}

By setting the determinant of the matrix $\Delta_{N}$ to zero, we obtain the transverse magnetic surface plasmon modes of the thin film. When the QE transition dipole moment orientation is along $z$, as it is assumed here, there is no excitation of the transverse electric modes, which, in general, have small impact. The thin film is placed between two $\mathrm{WS}_{2}$ layers; in addition, it is embedded in a constant dielectric permittivity medium $\varepsilon_{1}=\varepsilon_{3}$. Therefore the system of the nanostructure embedded in such a medium is a symmetric configuration, which supports two transverse magnetic modes, a symmetric and an antisymmetric one [51,52]. However, since we use real experimental values to describe the dielectric permittivity of the noble metals, the antisymmetric mode does not have strong contribution to the relaxation rate of the QE [52].

In Fig. 3 we present the dispersion relation $\hbar \omega\left(k_{\mathrm{SP}}\right)$ of an Au slab, of thickness of $2 \mathrm{~nm}$, for different values of the dielectric permittivity of the host medium, $\varepsilon_{1}=\varepsilon_{3}=1,4,8$. We compare the dispersion relation of the bare $\mathrm{Au}$ film with the case the Au thin film to be sandwiched between a pair of $\mathrm{WS}_{2}$ layers (green curves). The modes supported from the $\mathrm{WS}_{2}$ layers, which sandwich the Au thin film, can be probed by the energy loss probability spectrum of electrons penetrating the nanostructure; the energy loss probability is related to the value of the imaginary part of the Green's tensor, given by Eq. (7). In Fig. 3 we present normalized values of the quantity $\operatorname{Im}\left[d \mathfrak{G}(\mathbf{r}, \mathbf{r}, \omega) / d k_{S}\right]$. In the following analysis, we focus on understanding the modes supported by the $\mathrm{WS}_{2} / \mathrm{Au}$ slab/WS $\mathrm{W}_{2}$ nanostructure; therefore, the actual value of the energy loss probability is not of importance in our analysis in Fig. 3, where the bright color is connected with the SP mode.

When a bare $\mathrm{Au}$ film is considered, we observe that as the value of the dielectric permittivity of the host medium is increased, the surface plasmon dispersion relation is red shifted towards lower energies. Thus the dielectric permittivity of the host medium can provide us with a knob of tuning the dispersion of the plasmon modes; this property can be important in biosensor applications, where the surface plasmon sensitivity to the dielectric environment is crucial for detecting the right target material [53]. Moreover, by increasing the dielectric permittivity of the host medium the dispersion of the plasmon modes increases too, meaning that the SP modes are tightly confined to the metal dielectric interface. When we consider the full geometry, $\mathrm{WS}_{2} / \mathrm{Au}$ slab/ $\mathrm{WS}_{2}$, we observe that there is still some tunability through the dielectric permittivity of the host medium, although there is small difference in the dispersion relation between the host media dielectric permittivity of $\varepsilon_{1}=\varepsilon_{3}=1$ and $\varepsilon_{1}=\varepsilon_{3}=4$. The reason is that the $\mathrm{WS}_{2}$ layers provide the high refractive index component that has already red shifted the surface exciton-polariton branch. Moreover, we observe that there is an exciton-polariton mode at $2.02 \mathrm{eV}$, where the energy of the exciton $A$ of the $\mathrm{WS}_{2}$ lies, coupled with the plasmon mode provided by the metallic thin film. The higher the refractive index of the host medium of the $\mathrm{WS}_{2} / \mathrm{Au} / \mathrm{WS}_{2}$ system, the weaker the coupling between the exciton and the plasmon mode is, due to the red shift of the plasmon mode to lower energies, thus making their coupling off resonant.

In Fig. 4 we present the Purcell factor of a QE with zoriented transition dipole moment placed $2 \mathrm{~nm}$ away from a film of thickness $D=2 \mathrm{~nm}$, where we vary its emission energy $\hbar \omega$, as well as the value of the dielectric permittivity of the host medium. Different material arrangements are considered for the multilayer nanostructure. In Fig. 4(a) we present the Purcell factor of a $\mathrm{QE}$ above a pair of $\mathrm{WS}_{2}$ layers, which are $2 \mathrm{~nm}$ apart, while varying its emission energy $\hbar \omega$. We consider the $\mathrm{QE} /$ nanostructure embedded in homogeneous dielectric media, with $\varepsilon_{1}=\varepsilon_{2}=\varepsilon_{3}$. We 


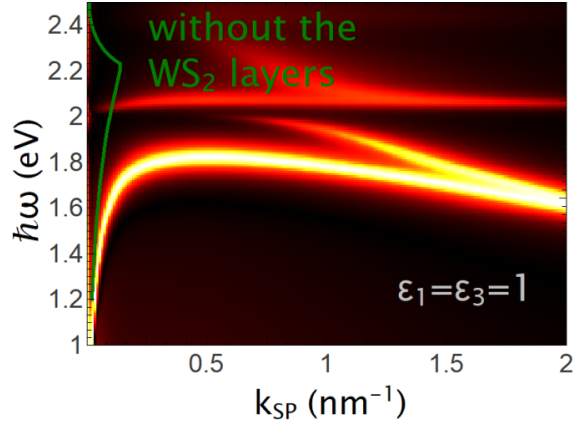

(a)

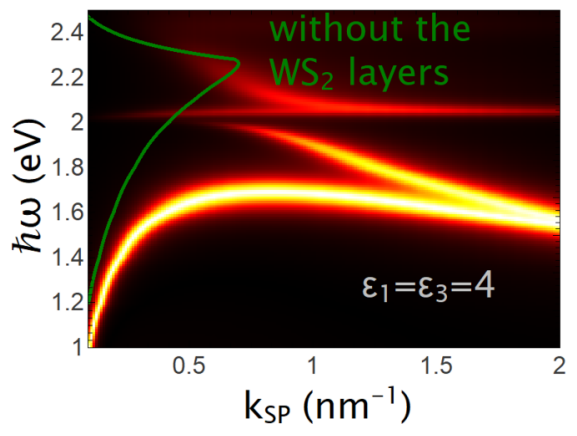

(b)

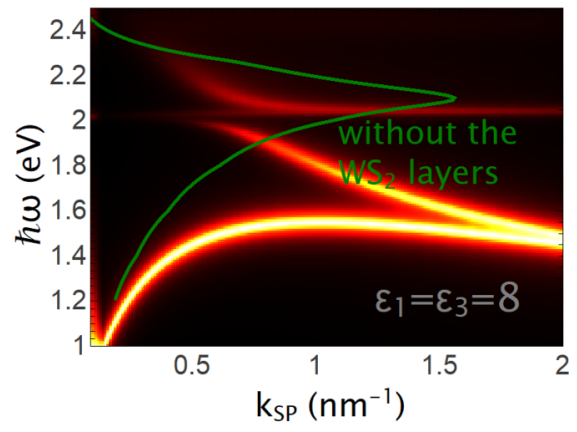

(c)

FIG. 3. Dispersion relation $\hbar \omega\left(k_{S P}\right)$ of the surface plasmon-polariton of a thin film of Au, with thickness $D=2 \mathrm{~nm}$, with and without (green curve) the two $\mathrm{WS}_{2}$ layers for various values of the dielectric permittivity of the host media: (a) $\varepsilon_{1}=\varepsilon_{3}=1$, (b) $\varepsilon_{1}=\varepsilon_{3}=4$, and (c) $\varepsilon_{1}=\varepsilon_{3}=8$.

observe that by increasing the value of the dielectric permittivity, the Purcell factor drops due to the dielectric screening of the host medium to the $\mathrm{WS}_{2}$ layers. Furthermore, we observe a sharp peak in the Purcell factor curve, which is related to the energy of the lower-energy exciton $A$ supported by the $\mathrm{WS}_{2}$ layer with energy $E_{A}=2.02 \mathrm{eV}$. Moreover, a broader peak is also present, related to the higher-energy excitons, which in the following analysis we omit. Our main focus will be at energies close to the exciton $A$ energy, $E_{A}=2.02 \mathrm{eV}$, corresponding to a wavelength of $\lambda_{A}=614 \mathrm{~nm}$.

In Fig. 4(b) we present the Purcell factor of a QE next to an Au slab of thickness $D=2 \mathrm{~nm}$. We observe a major broad peak in the Purcell factor, which is gradually red shifted as the value of the dielectric permittivity of the host media is increased. Furthermore, for the higher values of the dielectric permittivity, $\varepsilon_{1}=\varepsilon_{3}=8,12$, a second smaller peak in the Purcell factor curve, next to the major one, appears, due to the coupling with the two branches of the surface plasmon modes supported by the Au thin film layer. This fact is related to the penetration depth of the plasmon field, which is defined as $\delta_{\mathrm{SP}}=1 / 2 \operatorname{Im}\left(k_{z}^{\mathrm{SP}}\right)$, with $k_{z}^{\mathrm{SP}}=\sqrt{k_{1}^{2}-k_{\mathrm{SP}}^{2}} \simeq i k_{\mathrm{SP}}$. When the $\mathrm{QE}$ is placed at $z_{\mathrm{QE}}=2 \mathrm{~nm}$ away from the $\mathrm{Au}$ slab, it can efficiently couple to surface plasmon modes with $k_{\mathrm{SP}}=$ $0.5 \mathrm{~nm}^{-1}$, since for this value of the surface plasmon wave vector, as we realize from Fig. 3(a), they can only couple along the lower branch of the surface plasmon dispersion relation in case of the smaller values of the dielectric permittivity of the host media, $\varepsilon_{1}=\varepsilon_{3}=1$, 4 . Only for the higher values of the dielectric permittivity of the host media they can also couple along the upper polariton branch. The upper polariton branch supports modes that are not tightly confined; thus, they have a smaller contribution to the Purcell factor.

In Fig. 4(c) we present the Purcell factor of a QE placed $2 \mathrm{~nm}$ above a $\mathrm{WS}_{2} / \mathrm{Au} \mathrm{slab} / \mathrm{WS}_{2}$ nanostructure as a function of its emission energy, $\hbar \omega$ for an Au slab of $2 \mathrm{~nm}$ thickness. We observe that the Purcell factor has two peaks; this is due to the hybridization of the plasmon modes supported by the Au thin film with the exciton $A$ of the two $\mathrm{WS}_{2}$ layers. At emission energies close to the energy of the exciton $A$, $E_{A}=2.02 \mathrm{eV}$, the Purcell factor drops due to the energy transfer process from the $\mathrm{QE}$ to the electron-hole pairs in the $\mathrm{WS}_{2}$ layers. Furthermore, in Fig. 4(c) we observe that the Purcell factor peak corresponding to the lower-energy $\hbar \omega$, which is related to the symmetric mode, is red shifted and its value drops due to the dielectric screening, as the value of the dielectric permittivity of the host medium is increased. On the other hand, the position of the peak in the Purcell factor at higher-energy $\hbar \omega$, is only slightly red shifted as the value of the dielectric host media changes. This effect is due to the fact that this peak of the Purcell factor is related to

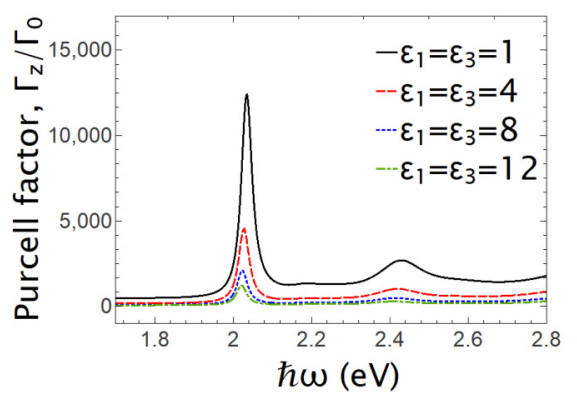

(a)

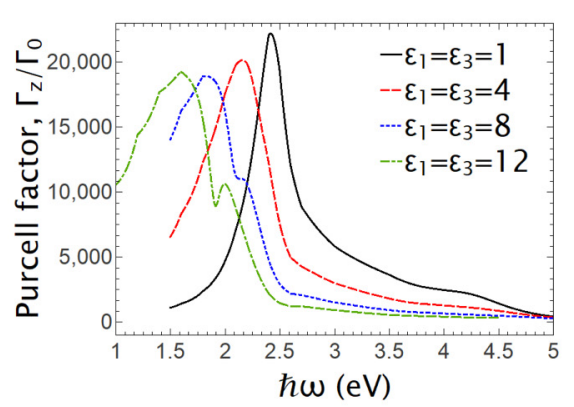

(b)

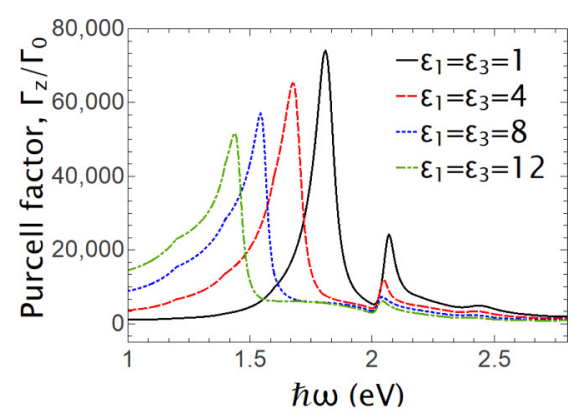

(c)

FIG. 4. The Purcell factor of a QE with a z-oriented transition dipole moment placed close to different QE/nanostructure configurations as function of the emission energy $\hbar \omega$ : (a) Two $\mathrm{WS}_{2}$ layers are considered on a homogeneous dielectric environment without the thin Au layer in between. (b) Au film of thickness $D=2 \mathrm{~nm}$ without the two $\mathrm{WS}_{2}$ layers. (c) Two $\mathrm{WS}_{2}$ layers with an Au film of thickness $D=2 \mathrm{~nm}$ in between. Various dielectric permittivity values of the host media are considered. 


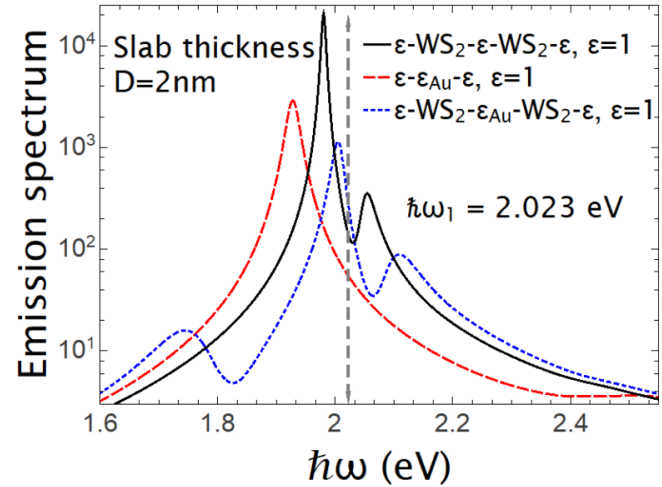

(a)

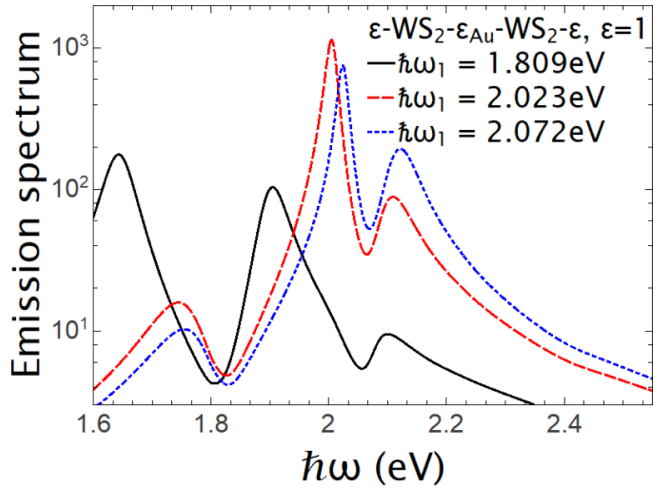

(b)

FIG. 5. The SE spectrum of a QE, placed $2 \mathrm{~nm}$ away from a three-layer nanostructure. Different three-layer nanostructure configurations and $\mathrm{QE}$ transitions energies are considered.

the excitons $A$ of the $\mathrm{WS}_{2}$ layers. However, the value of the Purcell factor at the higher-energy peak is decreased, as in the case of the lower-energy peak, due to the dielectric screening as the dielectric permittivity of the host media is increased.

We will now pay specific attention to the case with $\varepsilon_{1}=$ $\varepsilon_{3}=1$. In case of a pair of infinite $\mathrm{WS}_{2}$ layers we find that the highest Purcell factor value is at the energy of excitons $A, E_{A}=2.02 \mathrm{eV}$. When a single Au thin film of $D=2 \mathrm{~nm}$ is considered we observe a broad peak in the Purcell factor at the energy corresponding to the plasmon-polariton excitation, $\hbar \omega=2.45 \mathrm{eV}$. When finally the $\mathrm{WS}_{2} / \mathrm{Au}$ film $/ \mathrm{WS}_{2}$ nanostructure is considered, we observe two peaks in the Purcell factor due to the interaction between the surface plasmon mode supported by the Au thin film, and the excitons $A$ of the two $\mathrm{WS}_{2}$ layers. In case of the $\mathrm{WS}_{2} / \mathrm{Au}$ film/ $/ \mathrm{WS}_{2}$ nanostructure, the Purcell factor value exceeds $7 \times 10^{4}$, while in case of the single Au film the Purcell value is reduced to less than half, clearly showing that the $\mathrm{WS}_{2} / \mathrm{Au} \mathrm{slab} / \mathrm{WS}_{2}$ is suitable for strong enhancement of the light-matter interaction between the $\mathrm{QE}$ and the nanostructure.

In Fig. 5(a) we present the SE spectrum, using Eq. (9), of a QE placed $2 \mathrm{~nm}$ above the nanostructures studied in Fig. 4. From Eq. (7) it is clear that the coupling strength of the light-matter interaction between the QE and a nanostructure depends on the Purcell factor of the QE and the free-space decay rate of the QE; the higher their values, the stronger the coupling, in both cases. We note that larger free-space decay rates of the $\mathrm{QE}$ require smaller values of the Purcell factor in order to achieve strong light-matter coupling between a $\mathrm{QE}$ and a nanostructure. We consider a free-space decay width of $\hbar \Gamma_{0}\left(\omega_{1}\right)=60 \mu \mathrm{eV}$, which corresponds to typical molecules or dyes. Furthermore, we assume that the energy difference between the ground and the excited state of the QE matches the energy of the exciton $A, \hbar \omega_{1}=2.023 \mathrm{eV}$. In Fig. 5(a) we observe a very interesting behavior, namely when only the pair of the $\mathrm{WS}_{2}$ layers are present the QE emission spectrum splits into two peaks, which is the Rabi splitting, clearly presenting that the QE-nanostructure interaction is in the strong coupling regime. The $\mathrm{QE}$ coherently exchanges energy with the excitons $A$ of the $\mathrm{WS}_{2}$ layers. When the QE interacts only with the Au thin film a single peak is observed in the SE spectrum, which is displaced with respect to the emission energy of the $\mathrm{QE} \hbar \omega_{1}=2.023 \mathrm{eV}$ due to the interaction of the QE with the Au plasmon at $\hbar \omega=$ $2.45 \mathrm{eV}$, as indicated by the broad peak in the corresponding Purcell factor curve at this energy. In this case apparently the system operates in the weak coupling regime.

When the multilayer $\mathrm{WS}_{2} / \mathrm{Au}$ slab/WS 2 nanostructure is considered, we observe that the $\mathrm{SE}$ spectrum of the $\mathrm{QE}$ features a triplet of peaks, due to coupling of the $\mathrm{QE}$ with the lower and higher energy exciton-plasmon modes manifested in the Purcell factor spectrum. The energy difference between the two lower-energy peaks in the SE spectrum is $\hbar \omega_{R}=257 \mathrm{meV}$, clearly indicating that the light-matter interaction is in the strong coupling regime, or even close to the ultrastrong coupling regime [54], when one considers that the ratio of the Rabi splitting to the emission energy of the QE amounts to $\omega_{R} / \omega_{1}=13 \%$ here. Therefore, from Fig. 5(a) it is clear that the $\mathrm{WS}_{2} / \mathrm{Au}$ slab/WS nanostructure shows the most interesting effects and we focus on this case in Fig. 5(b).

In Fig. 5(b) we consider three different $\mathrm{QE}$ emission energies: $\hbar \omega_{1}=1.809 \mathrm{eV}$ and $\hbar \omega_{1}=2.072 \mathrm{eV}$, which match the positions of the two peaks of the Purcell factor in Fig. 4(c), and $\hbar \omega_{1}=2.023 \mathrm{eV}$, which matches the exciton $A$ energy of the $\mathrm{WS}_{2}$ layers. We now observe three peaks in the SE spectrum of the $\mathrm{QE}$ at these emission energies considered. In particular, when the emission energy is $\hbar \omega_{1}=1.809 \mathrm{eV}$, the Rabi splitting is $\hbar \omega_{R}=0.261 \mathrm{eV}$ and the ratio $\omega_{R} / \omega_{1}$ amounts to $14.4 \%$, an indication that the light-matter interaction strength enters the ultrastrong coupling regime.

The dispersion relation, $\hbar \omega\left(k_{\mathrm{SP}}\right)$, of the surface plasmon mode supported by a thin film of $\mathrm{Ag}$, with thickness of $D=$ $2 \mathrm{~nm}$, is presented in Fig. 6(a). We observe similar behavior for the dispersion relation to the case when we consider a $\mathrm{Au}$ thin film in Fig. 3(a). We find that as the value of the dielectric permittivity increases, the surface plasmon dispersion relation of the Ag film is red shifted. When the Ag thin film is between a pair of $\mathrm{WS}_{2}$ layers, the surface plasmons of the $\mathrm{Ag}$ thin film and the excitons $A$ of the $\mathrm{WS}_{2}$ layers interact resulting in hybridized modes. The hybridization is close to the exciton $A$ energy $\hbar \omega_{A}=2.02 \mathrm{eV}$, where in case of a dielectric permittivity $\varepsilon_{1}=\varepsilon_{3}=1$ it emerges as a pronounced splitting. As the value of the dielectric permittivity $\varepsilon_{1}=\varepsilon_{3}$ increases, 


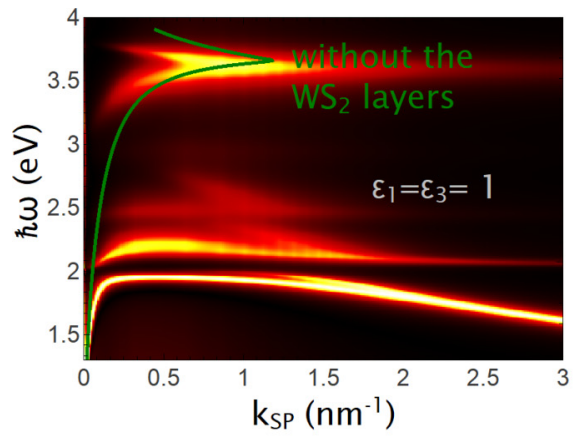

(a)

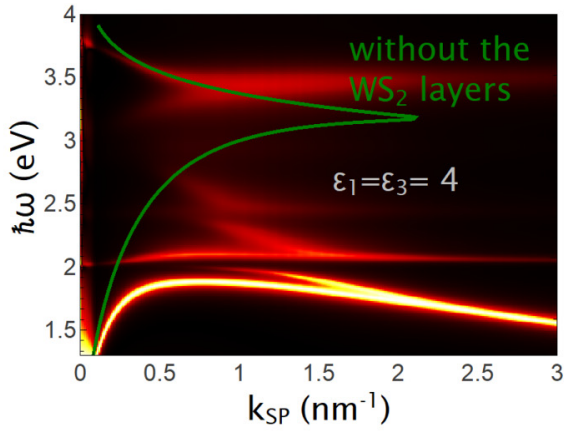

(b)

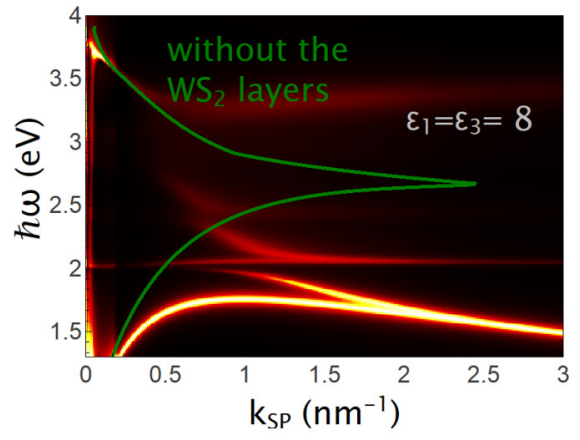

(c)

FIG. 6. Dispersion relation $\hbar \omega\left(k_{S P}\right)$ of the surface plasmon polariton of a thin film of $\mathrm{Ag}$, thickness $D=2 \mathrm{~nm}$, when the dielectric permittivity of the host media changes: (a) the case without $\mathrm{WS}_{2}$ layers, (b), (c) the cases with and without (green curve) WS $\mathrm{Wlyers}_{2}$ for the dielectric permittivity of the host medium $\varepsilon_{1}=\varepsilon_{2}=4$ and 8 .

the coupling between the exciton and the plasmon is reduced; this behavior is attributed to the dielectric screening caused by the higher dielectric value of the host medium. We note two differences between the dispersion relation for the $\mathrm{Ag}$ film, as presented in this figure, and the corresponding results for the Au film shown in Fig. 3: first, the surface plasmon mode dispersion relations for the $\mathrm{Ag}$ are blue shifted, compared to the $\mathrm{Au}$, and second, the $\mathrm{Ag}$ dispersion relation is more dispersive than the Au case due to the lower material losses.

In Fig. 7(a) we present the Purcell factor of a QE placed $2 \mathrm{~nm}$ away from a $\mathrm{Ag}$ thin film, which has a thickness of $D=2 \mathrm{~nm}$, varying its emission energy. Since we anticipate similar dependence of the Purcell factor of a QE as the dielectric permittivity of the host medium is increased, for the case of $\mathrm{Ag}$ to $\mathrm{Au}$ nanostructures, we now focus when the dielectric environment is the free space, $\varepsilon_{1}=\varepsilon_{3}=1$. We find that for the single Ag thin film, the Purcell factor has a single peak due to the excitation of the surface plasmon mode. In the case of the $\mathrm{WS}_{2} / \mathrm{Ag}$ film/ $\mathrm{WS}_{2}$ nanostructure, the Purcell factor shows two peaks due to the creation of exciton-plasmon hybrid modes. Although the Purcell factor value of the QE is smaller for the case of the Ag nanostructure compared to the $\mathrm{Au}$. The reason for this is that the material losses of $\mathrm{Au}$ are smaller than Ag at low energies.
We continue in Fig. 7(b) by discussing the SE spectrum of a QE, placed $2 \mathrm{~nm}$ above the multilayer nanostructures. The emission energy of the QE is $\hbar \omega_{1}=2.023 \mathrm{~nm}$, matching the exciton $A$ energy $E_{A}$ of the $\mathrm{WS}_{2}$ layers. The largest Rabi splitting is observed for the $\mathrm{WS}_{2} / \mathrm{Ag}$ film/WS nanostructure, and has the value of $\hbar \omega_{R}=125 \mathrm{meV}$; in this case, the ratio $\omega_{R} / \omega_{1}=6.1 \%$. Now the splitting is less pronounced when compared to the Au case. However, we have to remember that due to the $\mathrm{WS}_{2}$ layers, the surface plasmon modes hybridize at energies close to the exciton energy $E_{A}$ where the imaginary part of the dielectric permittivity of $\mathrm{Au}$ is smaller than the corresponding values for Ag, as shown in Fig. 2(b). We note that when the bare Ag film is considered there is no splitting in the emission spectrum of the $\mathrm{QE}$, since the light-matter interaction strength between the $\mathrm{QE}$ and the nanostructure is within the weak coupling limit.

In Fig. 7(c) we present the SE spectrum of a QE placed $2 \mathrm{~nm}$ away from the $\mathrm{WS}_{2} / \mathrm{Ag}$ film/WS $\mathrm{W}_{2}$ nanostructure, with Ag film thickness $D=2 \mathrm{~nm}$, considering three emission energies, $\hbar \omega_{1}=1.950 \mathrm{eV}$ and $\hbar \omega_{1}=2.166 \mathrm{eV}$, which correspond to the two peaks of the Purcell factor of the QE as shown in Fig. 7(b), and $\hbar \omega_{1}=2.023 \mathrm{eV}$, which matches the exciton $A$ energy $E_{A}$ of the $\mathrm{WS}_{2}$ layers. We observe that at all emission energies considered, three peaks are observed

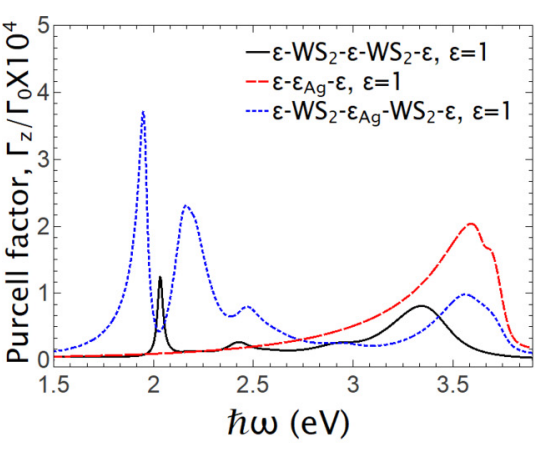

(a)

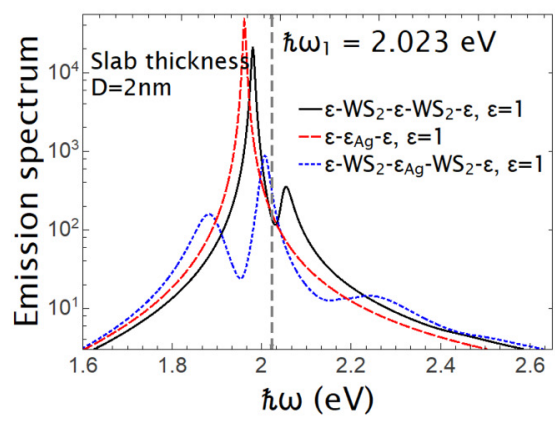

(b)

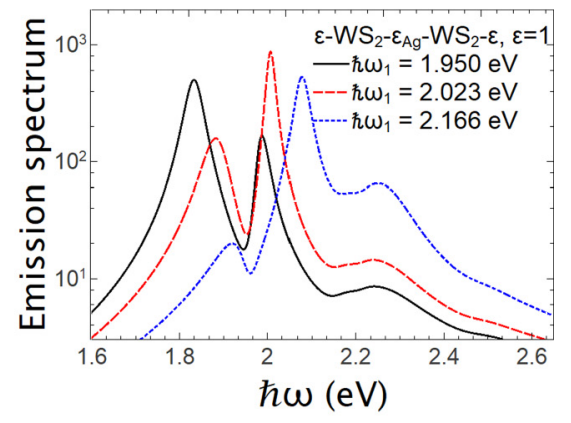

(c)

FIG. 7. (a) Purcell factor of a QE, placed $2 \mathrm{~nm}$ away of the three-layer nanostructure configurations with an Ag thin film, for a host medium dielectric permittivity of $\varepsilon=\varepsilon_{1}=\varepsilon_{3}=1$. (b), (c) The emission spectrum of a QE, placed $2 \mathrm{~nm}$ away from a three-layer nanostructure with an Ag thin film. Different three-layer nanostructure configurations and QE transitions energies are considered. 


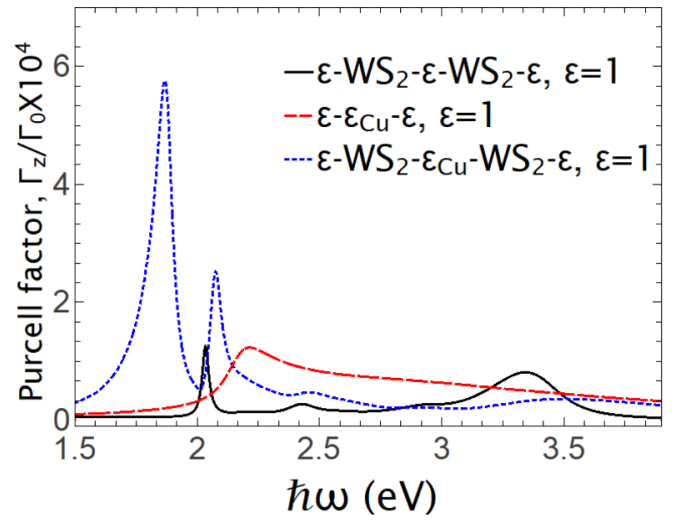

(a)

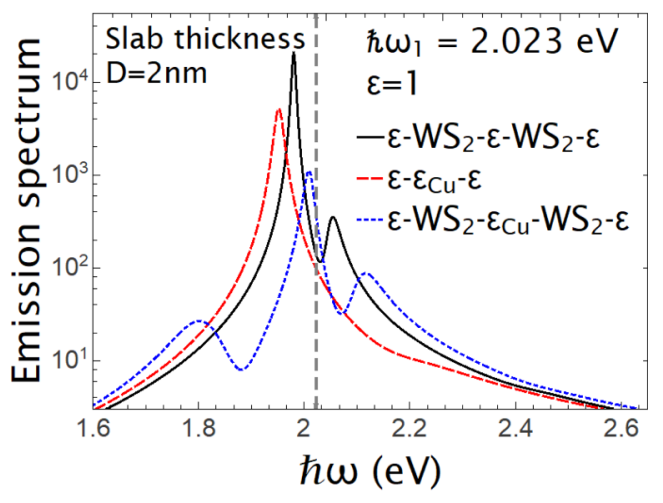

(b)

FIG. 8. (a) Purcell factor of a QE, placed $2 \mathrm{~nm}$ away of the three-layer nanostructure configurations with a $\mathrm{Cu}$ thin film, for a host medium dielectric permittivity of $\varepsilon=\varepsilon_{1}=\varepsilon_{3}=1$. (b) The emission spectrum of a QE, placed $2 \mathrm{~nm}$ away from a three-layer nanostructure with a Cu thin film for different three-layer nanostructure configurations.

in the SE spectrum of the QE. The fact that they are three peaks is related to the overlap of the splittings of the Purcell factor peaks in Fig. 7(b). The largest Rabi splitting value is observed when the emission energy is $\hbar \omega_{1}=1.950 \mathrm{eV}$ and the splitting between the two lower energy peaks in the SE spectrum of the $\mathrm{QE}$ amounts to $\hbar \omega_{R}=0.156 \mathrm{eV}$, resulting into $\omega_{R} / \omega_{1}=8 \%$. The Rabi splittings presented are a clear sign that the light-matter interaction strength between the $\mathrm{QE}$ and the nanostructure lies in the strong coupling regime here.

In the following we present results regarding two other noble metals, namely $\mathrm{Cu}$ and $\mathrm{Al}$, that their optical properties in relation to the $\mathrm{SE}$ spectrum of a $\mathrm{QE}$ in proximity to multilayer nanostructures including these metals are relatively less explored than in case of the noble metals $\mathrm{Au}$ and $\mathrm{Ag}$. In Figs. 8(a) and 9(a) we present the Purcell factor of a QE placed $2 \mathrm{~nm}$ away from a thin film of $\mathrm{Cu}$ and $\mathrm{Al}$, respectively, which in both cases has a thickness of $D=2 \mathrm{~nm}$, varying its emission energy. We compare the Purcell factor of a $\mathrm{QE}$ interacting with two $\mathrm{WS}_{2}$ layers, a bare $\mathrm{Cu}$ or $\mathrm{Al}$ film and the $\mathrm{WS}_{2} / \mathrm{Cu}$ or $\mathrm{Al}$ film/ $/ \mathrm{WS}_{2}$ nanostructures. Similar results to the
$\mathrm{Au}$ and $\mathrm{Ag}$ thin film results presented above are observed for the $\mathrm{Al}$ and $\mathrm{Cu}$ thin film cases. We note that for the $\mathrm{Al}$ thin film smaller values of the Purcell factor are observed, compared to the $\mathrm{Au}, \mathrm{Ag}$, and $\mathrm{Cu}$ cases, due to higher material losses. It is thus expected that for $\mathrm{Al}$ strong coupling effects will be less profound, which will make itself apparent in the SE spectrum of the QE in this case.

In Figs. 8(b) and 9(b), we present the SE spectrum of a QE, placed $2 \mathrm{~nm}$ above the multilayer nanostructures including the $\mathrm{Cu}$ and $\mathrm{Al}$ thin films, respectively. The transition energy of the $\mathrm{QE}$ is $\hbar \omega_{1}=2.023 \mathrm{eV}$, matching the exciton $A$ energy $E_{A}$ of the $\mathrm{WS}_{2}$ layers. The largest Rabi splitting is observed for $\mathrm{WS}_{2} / \mathrm{Cu}$ film/WS $/ \mathrm{WS}_{2}$ nanostructure, and has the value of $\hbar \omega_{R}=208 \mathrm{meV}$, with $\omega_{R} / \omega_{1}=10.3 \%$. We note that the Rabi splitting for the $\mathrm{Cu}$ is larger than in the $\mathrm{Ag}$ case; thus, our analysis shows that $\mathrm{Cu}$ should work better than $\mathrm{Ag}$ for applications such as single photon emitters and related quantum technologies. For the Al case considered in Fig. 9(b), we find that the SE spectrum of the $\mathrm{QE}$ in the energy region of the exciton $A$ of the $\mathrm{WS}_{2}$ is in the weak coupling regime, since no splitting is observed.

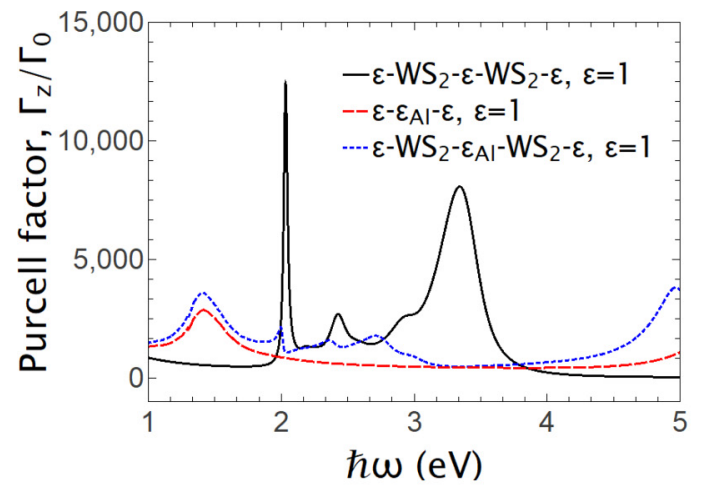

(a)

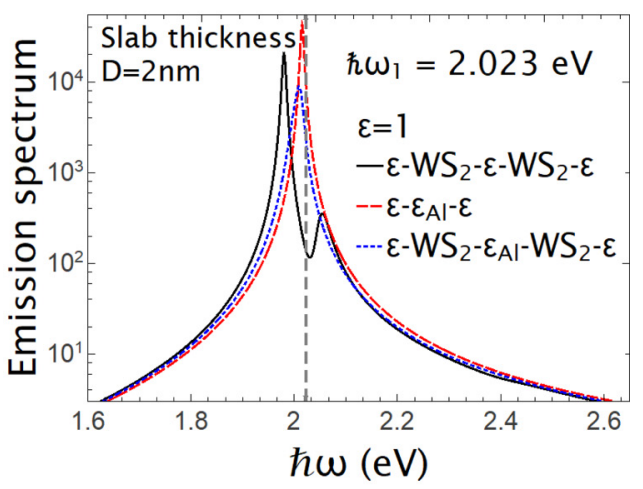

(b)

FIG. 9. (a) Purcell factor of a QE, placed $2 \mathrm{~nm}$ away of the three-layer nanostructure configurations with an $\mathrm{Al}$ thin film, for a host medium dielectric permittivity of $\varepsilon=\varepsilon_{1}=\varepsilon_{3}=1$. (b) The emission spectrum of a QE, placed $2 \mathrm{~nm}$ away from a three-layer nanostructure with an Al thin film for different three-layer nanostructure configurations. 


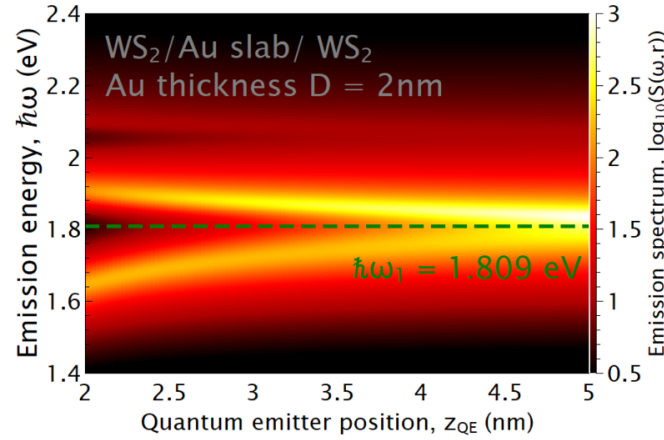

(a)

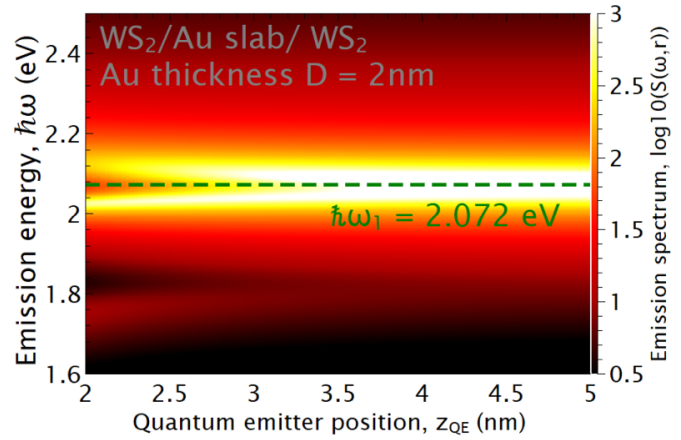

(b)

FIG. 10. Contour plot of the logarithm of the emission spectrum, $\log _{10}(S(\mathbf{r}, \omega))$, of a QE interacting with a $\mathrm{WS}_{2} / \mathrm{Au}$ film/WS 2 structure, for varying the QE position away from the nanostructure, $z_{\mathrm{QE}}$, and its emission energy, $\hbar \omega$. Two transition energies of the QE are considered: (a) $\hbar \omega=1.809 \mathrm{eV}$ and (b) $\hbar \omega=2.072 \mathrm{eV}$. The Au thin film thickness is $D=2 \mathrm{~nm}$.

From Eq. (9) it is apparent that the SE spectrum of the QE strongly depends on the Purcell factor, where the higher values of the Purcell factor lead to higher values of the Rabi splitting. On its turn, the Purcell factor of the QE depends on the separation distance between the $\mathrm{QE}$ and the multilayer nanostructure discussed here. When the transition energy of the $\mathrm{QE}, \hbar \omega_{1}$, is close to the exciton $A$ energy of the $\mathrm{WS}_{2}$, then the Purcell factor follows a power-law distance dependence reduction. However, when the transition energy of the QE is close to the plasmon energy, then its Purcell factor follows an exponential decay as the QE-nanostructure distance increases. The power-law dependence leads to a faster relaxation of the Purcell factor of the QE compared to the case of an exponential decay, for more details, see Ref. [19].

In Fig. 10 we present the contour plot of the logarithm of the SE spectrum, $\log _{10}(S(\mathbf{r}, \omega))$, of a QE interacting with the $\mathrm{WS}_{2} / \mathrm{Au}$ slab/WS structure, for varying the $\mathrm{QE}$ position away from the nanostructure, $z_{\mathrm{QE}}$, and its emission energy, $\hbar \omega$. The Au slab thickness is $D=2 \mathrm{~nm}$. We consider two transition energies of the QE $\hbar \omega=1.809 \mathrm{eV}$ for Fig. 10(a) and $\hbar \omega=2.072 \mathrm{eV}$ for Fig. 10(b), which are the energies matching the peaks of the Purcell factor of the QE in Fig. 4(c). In both panels in Fig. 10, we observe that as the separation distance between the $\mathrm{QE}$ and the $\mathrm{WS}_{2} / \mathrm{Au}$ slab/ $\mathrm{WS}_{2}$ structure is increased the emission spectrum goes from showing a clear Rabi splitting to a single emission peak.

At the separation distance of $z_{\mathrm{QE}}=5 \mathrm{~nm}$ the emission spectrum looks like a Gaussian spectrum with its peak value at $\hbar \omega_{1}$ of the QE. At $z_{\mathrm{QE}}=2 \mathrm{~nm}$ we observe, for both transition energies, the three peaks in the emission spectrum that we discussed in Fig. 5. Furthermore, we find that the initial splitting at the transition energy of the QE disappears faster for the case the transition energy is on resonance with the higher energy peak of the Purcell factor observed in Fig. 4(c), due to the exciton $A$ energy, $\hbar \omega_{1}=2.072 \mathrm{eV}$, as the separation distance increases. At separation distance of $z_{\mathrm{QE}}=2.5 \mathrm{~nm}$ this splitting has almost vanished. While, for the transition energy $\hbar \omega_{1}=1.809 \mathrm{eV}$, the splitting disappears for distances around $z_{\mathrm{QE}}=4.5 \mathrm{~nm}$. This behavior is connected with the exponential dependence reduction of the Purcell factor for the case the QE relaxes by exciting a plasmon mode compared to the case of the power-law relaxation for the case that the excitons in the $\mathrm{WS}_{2}$ layer are excited. The distance dependence of the emission spectrum is also important for spectroscopic and biosensor applications and its understanding is crucial for designing future practical applications.

\section{CONCLUSIONS}

In this work we presented a study of the SE of a $\mathrm{QE}$ placed close to a pair of $\mathrm{WS}_{2}$ layers separated by a thin film of either a dielectric or a noble metal. When the $\mathrm{QE}$ is located close to the $\mathrm{WS}_{2} /$ dielectric or noble metal layer/WS nanostructure, large values of the Purcell factor are obtained at energies close to the exciton $A$ energy, $\hbar \omega_{A}=2.02 \mathrm{eV}$, which is supported by the $\mathrm{WS}_{2}$ layers, as well as at lower energies, which are close to the modified plasmon-exciton resonance. We also found that the exciton $A$ of the $\mathrm{WS}_{2}$ layers and the surface plasmon modes supported by the thin metallic film are hybridized, as shown in the dispersion relations presented.

The metal thin film considered was composed of the noble metals $\mathrm{Au}, \mathrm{Ag}, \mathrm{Cu}$, and $\mathrm{Al}$. At energies close to the $\mathrm{WS}_{2}$ exciton $A$ energy, $\mathrm{Al}$ has a poor performance, while $\mathrm{Au}, \mathrm{Ag}$, and $\mathrm{Cu}$ have substantially better performance in enhancing the light-matter interaction between the $\mathrm{QE}$ and the nanostructure, since the smaller material losses of the $\mathrm{Au}, \mathrm{Ag}$, and $\mathrm{Cu}$ allow for attaining strong, or even ultrastrong coupling between the $\mathrm{QE}$ and the multilayer structure. The form of the SE spectrum of the QE next to the $\mathrm{WS}_{2} /$ metal film/WS nanostructure, in case of a $\mathrm{QE}$ with transition energy matching the exciton energy $A$ or the energy at the peaks of the Purcell factor, indicates clearly the light-matter interaction strength between the QE and the nanostructure. More specifically, when the light-matter interaction strength between the QE and the $\mathrm{WS}_{2}$ /noble metal layer/ $\mathrm{WS}_{2}$ multilayer lies in the weak coupling regime, a single emission peak appears, while in case of the strong coupling regime, bordering, in certain cases, to the ultrastrong coupling regime, indicated by the large values of Rabi splitting observed, three emission peaks are present, due to the existence of two peaks in the Purcell factor. Interestingly, we also found that $\mathrm{Cu}$ is a material that shows strong optical response similarly to the $\mathrm{Au}$, and distinctly stronger than Ag. 
We further stress that the optical properties of a QE next to a $\mathrm{WS}_{2} /$ noble metal layer/ $\mathrm{WS}_{2}$ nanostructure can be tuned by changing the dielectric permittivity of the host media; a property that could be of importance in biosensor applications, where the emission spectrum is used as a fingerprint of the target material usually emitting in the visible part of the spectrum. Moreover, the TMD materials can be functionalized with carbon layers, which is a biocompatible material, for increasing their applicability. Lastly, we also stress that our results were obtained by using dielectric per- mittivities extracted from experiments, which makes our conclusions particularly prone to currently feasible experimental investigations.

\section{ACKNOWLEDGMENTS}

The contribution of E.P. is cofinanced by Greece and the European Union-European Regional Development Fund via the General Secretariat for Research and Technology (GSRT) (Project POLISIMULATOR).
[1] W. L. Barnes, J. Opt. A 8, S87 (2006).

[2] D. G. Baranov, M. Wersäll, J. Cuadra, T. J. Antosiewicz, and T. Shegai, ACS Photon. 5, 24 (2018).

[3] M. Pelton, S. D. Storm, and H. Leng, Nanoscale 11, 14540 (2019).

[4] P. A. D. Goncalves, N. Stenger, J. D. Cox, N. Asger Mortensen, and S. Xiao, Adv. Opt. Mater. 8, 1901473 (2020).

[5] M. S. Tame, K. R. McEnery, S. K. Özdemir, J. Lee, S. A. Maier, and M. S. Kim, Nature Phys. 9, 329 (2013).

[6] B. Kolaric, B. Maes, K. Clays, T. Durt, and Y. Caudano, Adv. Quant. Technol. 1, 1800001 (2018).

[7] S. I. Bogdanov, A. Boltasseva, and V. M. Shalaev, Science 364, 532 (2019).

[8] M. Gurioli, Z. Wang, A. Rastelli, T. Kuroda, and S. Sanguinetti, Nature Mater. 18, 799 (2019).

[9] G. M. Akselrod, C. Argyropoulos, T. B. Hoang, C. Ciraci, C. Fang, J. Huang, D. R. Smith, and M. H. Mikkelsen, Nature Photon. 8, 835 (2014).

[10] T. B. Hoang, G. M. Akselrod, C. Argyropoulos, J. Huang, D. R. Smith, and M. H. Mikkelsen, Nature Commun. 6, 7788 (2015).

[11] J. B. Khurgin, Nature Nanotechnol. 10, 2 (2015).

[12] R. A. Maniyara, D. Rodrigo, R. Yu, J. Canet-Ferrer, D. S. Ghosh, R. Yongsunthon, D. E. Baker, A. Rezikyan, F. J. Garcia de Abajo, and V. Pruneri, Nature Photon. 13, 328 (2019).

[13] Z. M. Abd El-Fattah, V. Mkhitaryan, J. Brede, L. Fernández, C. Li, Q. Guo, A. Ghosh, A. R. Echarri, D. Naveh, F. Xia, J. E. Ortega, and F. J. García de Abajo, ACS Nano 13, 7771 (2019).

[14] L. Britnell, R. M. Ribeiro, A. Eckmann, R. Jalil, B. D. Belle, A. Mishchenko, Y.-J. Kim, R. V. Gorbachev, T. Georgiou, S. V. Morozov, A. N. Grigorenko, A. K. Geim, C. Casiraghi, A. H. C. Neto, and K. S. Novoselov, Science 340, 1311 (2013).

[15] F. Xia, H. Wang, D. Xiao, M. Dubey, and A. Ramasubramaniam, Nature Photon. 8, 899 (2014).

[16] K. F. Mak and J. Shan, Nature Photon. 10, 216 (2016).

[17] J. N. S. Gomes, C. Trallero-Giner, N. M. R. Peresl, and M. I. Vasilevskiy, J. Appl. Phys. 127, 133101 (2020).

[18] D. N. Basov, M. M. Fogler, and F. J. G. de Abajo, Science 354, 195 (2016).

[19] V. D. Karanikolas, C. A. Marocico, P. R. Eastham, and A. L. Bradley, Phys. Rev. B 94, 195418 (2016).

[20] C. Van Vlack, P. T. Kristensen, and S. Hughes, Phys. Rev. B 85, 075303 (2012).

[21] A. Gonzalez-Tudela, P. A. Huidobro, L. Martin-Moreno, C. Tejedor, and F. J. Garcia-Vidal, Phys. Rev. B 89, 041402(R) (2014).
[22] J. Hakami, L. Wang, and M. S. Zubairy, Phys. Rev. A 89, 053835 (2014).

[23] H. Varguet, B. Rousseaux, D. Dzsotjan, H. R. Jauslin, S. Guérin, and G. Colas des Francs, Opt. Lett. 41, 4480 (2016).

[24] R.-Q. Li, D. Hernangomez-Perez, F. J. Garcia-Vidal, and A. I. Fernandez-Dominguez, Phys. Rev. Lett. 117, 107401 (2016).

[25] I. Thanopulos, V. Yannopapas, and E. Paspalakis, Phys. Rev. B 95, 075412 (2017).

[26] C.-J. Yang and J.-H. An, Phys. Rev. B 95, 161408(R) (2017).

[27] N. Iliopoulos, I. Thanopulos, V. Yannopapas, and E. Paspalakis, Phys. Rev. B 97, 115402 (2018).

[28] T. Neuman, R. Esteban, D. Casanova, F. J. Garcia-Vidal, and J. Aizpurua, Nano Lett. 18, 2358 (2018).

[29] B. Rousseaux, D. G. Baranov, M. Käll, T. Shegai, and G. Johansson, Phys. Rev. B 98, 045435 (2018).

[30] F. H. L. Koppens, D. E. Chang, and F. J.García de Abajo, Nano Lett. 11, 3370 (2011).

[31] L. Gaudreau, K. J. Tielrooij, G. E. D. K. Prawiroatmodjo, J. Osmond, F. J. G. de Abajo, and F. H. L. Koppens, Nano Lett. 13, 2030 (2013).

[32] L. Sun, B. Tang, and C. Jiang, Opt. Express 22, 26487 (2014).

[33] K. J. Tielrooij, L. Orona, A. Ferrier, M. Badioli, G. Navickaite, S. Coop, S. Nanot, B. Kalinic, T. Cesca, L. Gaudreau, Q. Ma, A. Centeno, A. Pesquera, A. Zurutuza, H. de Riedmatten, P. Goldner, F. J. García de Abajo, P. Jarillo-Herrero, and F. H. L. Koppens, Nature Phys. 11, 281 (2015).

[34] V. D. Karanikolas, C. A. Marocico, and A. L. Bradley, Phys. Rev. B 93, 035426 (2016).

[35] V. Karanikolas and E. Paspalakis, J. Phys. Chem. C 122, 14788 (2018).

[36] V. Karanikolas, P. Tozman, and E. Paspalakis, Phys. Rev. B 100, 245403 (2019).

[37] I. V. Bondarev and B. Vlahovic, Phys. Rev. B 75, 033402 (2007).

[38] M. Cuevas, J. Quant. Spectr Radiat. Trans. 214, 8 (2018).

[39] V. D. Karanikolas and E. Paspalakis, Phys. Rev. B 96, 041404(R) (2017).

[40] V. Karanikolas, I. Thanopulos, and E. Paspalakis, Opt. Lett. 44, 2049 (2019).

[41] I. Thanopulos, V. Karanikolas, and E. Paspalakis, Opt. Lett. 44, 3510 (2019).

[42] Y. Li, A. Chernikov, X. Zhang, A. Rigosi, H. M. Hill, A. M. van der Zande, D. A. Chenet, E.-M. Shih, J. Hone, and T. F. Heinz, Phys. Rev. B 90, 205422 (2014). 
[43] P. A. D. Goncalves, L. P. Bertelsen, S. Xiao, and N. A. Mortensen, Phys. Rev. B 97, 041402(R) (2018).

[44] G. T. Papadakis, A. Davoyan, P. Yeh, and H. A. Atwater, Phys. Rev. Materials 3, 015202 (2019).

[45] P. B. Johnson and R. W. Christy, Phys. Rev. B 6, 4370 (1972).

[46] K. M. McPeak, S. V. Jayanti, S. J. P. Kress, S. Meyer, S. Iotti, A. Rossinelli, and D. J. Norris, ACS Photonics 2, 326 (2015).

[47] H. T. Dung, L. Knöll, and D.-G. Welsch, Phys. Rev. A 62, 053804 (2000).

[48] A. Manjavacas, F. J. García de Abajo, and P. Nordlander, Nano Lett. 11, 2318 (2011).
[49] C. T. Tai, Dyadic Green Functions in Electromagnetic Theory (Oxford University Press, Oxford, 1994).

[50] W. C. Chew, Waves and Fields in Inhomogeneous Media (IEEE Press, New York, 1995).

[51] C. A. Marocico and J. Knoester, Phys. Rev. A 84, 053824 (2011).

[52] J. A. Dionne, L. A. Sweatlock, H. A. Atwater, and A. Polman, Phys. Rev. B 72, 075405 (2005).

[53] A. G. Brolo, Nature Photon. 6, 709 (2012).

[54] A. Frisk Kockum, A. Miranowicz, S. De Liberato, S. Savasta, and F. Nori, Nature Rev. Phys. 1, 19 (2019). 